\title{
Effect of Access to After-Hours Primary Care on the Association Between Home Nursing Visits and Same-Day Emergency Department Use
}

Aaron Jones, MSc, $P b D^{1}$

Susan E. Bronskill, $P b D^{2,3}$

Connie Scbumacher, RN, $P b D^{1}$

Hsien Seow, $P b D^{1,4}$

David Feeny, $\mathrm{PbD}^{5}$

Andrew P. Costa, PbD ${ }^{1,6}$

'Department of Health Research Methods, Evidence, and Impact, McMaster University, Hamilton, Ontario, Canada

${ }^{2}$ ICES, Toronto, Ontario, Canada

${ }^{3}$ Institute of Health Policy, Management \& Evaluation, Dalla Lana School of Public Health, University of Toronto

${ }^{4}$ Department of Oncology, McMaster University, Hamilton, Ontario, Canada

${ }^{5}$ Department of Economics, McMaster University, Hamilton, Ontario, Canada

${ }^{6}$ Department of Medicine, McMaster University, Hamilton, Ontario, Canada

Conflicts of interest: authors report none.

\section{CORRESPONDING AUTHOR}

Aaron Jones, MSc, PhD

Department of Health Research Methods, Evidence, and Impact

McMaster University

1280 Main St W

Hamilton, L8S 4K1

jonesa13@mcmaster.ca

\begin{abstract}
PURPOSE Previous work has demonstrated that home care patients have an increased risk of visiting the emergency department after a home nursing visit on the same day. We investigated whether this association is modified by greater access to after-hours primary care.

METHODS We conducted a population-based case-crossover study of home care patients in Ontario, Canada in 2014-2016. Emergency department visits after 5:00 PM were selected as case periods and matched, within the same patient, to control periods within the previous week. The association between home nursing visits and same-day emergency department visits was estimated with conditional logistic regression. Access to after-hours primary care, measured on the patient and practice level, was tested for effect modification using an interaction term approach. Analysis was performed separately for all emergency department visits and a less urgent subset not admitted to hospital.
\end{abstract}

RESULTS A total of 11,840 patients contributed cases to the analysis. Patients with a history of after-hours primary care use had a smaller increased risk of a sameday after-hours emergency department visit $(\mathrm{OR}=1.18 ; 95 \% \mathrm{Cl}, 1.06-1.30)$ compared with patients with no after-hours care $(\mathrm{OR}=1.31 ; 95 \% \mathrm{Cl}, 1.25-1.39)$. The modifying effect was stronger among emergency department visits not admitted to hospital $(\mathrm{OR}=1.11 ; 95 \% \mathrm{Cl}, 0.97-1.28$ vs $\mathrm{OR}=1.41 ; 95 \% \mathrm{Cl}, 1.31-1.51)$.

CONCLUSION Greater access to after-hours primary care reduced the risk of lessurgent emergency department use associated with home nursing visits. These findings suggest increasing access to after-hours primary care could prevent some less-urgent emergency department visits.

Ann Fam Med 2020;18:406-412. https://doi.org/10.1370/afm.2571.

\section{INTRODUCTION}

I $\mathrm{n}$ recent decades, health systems around the world have sought to increase access to primary care outside of traditional business hours. In theory, greater access to after-hours primary care can divert less-urgent visits away from the emergency department, which is crowded, disrupts continuity of care, and is typically not designed to meet the care needs of complex, older adults. ${ }^{1,2}$ The existing literature testing this hypothesis, however, has produced conflicting results, with some studies showing that increased access to after-hours primary care is associated with fewer emergency department visits ${ }^{3,4}$ and others finding no difference. ${ }^{5,6}$

Older adults receiving home care services are a growing, frail population with particularly high rates of emergency department use., Previous research has found that home nursing patients in Ontario, Canada have an increased risk of visiting the emergency department after 5:00 PM on the same day they receive a home nursing visit. ${ }^{9}$ This effect is presumed to be the result of the nurse identifying a health issue that they are unable to appropriately address during the visit and referring the patient for further care. Home care nursing in Ontario operates within a task-focused, 
visit-oriented model that limits a nurse's flexibility to move beyond prearranged tasks, hindering comprehensive practice, and likely contributing to the increased risk of a same-day emergency department visit. ${ }^{10}$ The increased risk was considerably higher for less-urgent emergency department visits, suggesting that many of the same-day visits could be more appropriately seen in a primary care setting. For visits of this nature, increased access to after-hours primary care could reduce the risk of a same-day emergency department visit.

Our objective was to examine whether greater access to after-hours primary care modifies the association between nursing visits and same-day emergency department visits. We hypothesized that increased access to after-hours primary care, measured at both the patient and provider level, would reduce the likelihood that a patient would visit the emergency department on the same day as a home nursing visit, and that the modification would be stronger for less-urgent emergency department visits.

\section{METHODS}

\section{Study Design and Data Sources}

This study extends a smaller case-crossover study previously conducted by our team. ${ }^{9}$ In the current study, we identified a population-based, retrospective cohort of publicly funded adult, home care patients in Ontario, Canada using multiple, linked, health administrative databases (Supplemental Appendix 1, https://www.Ann FamMed.org/content/18/5/406/suppl/DC1/). Home care patients and services were identified through the Home Care Database, which captures client and service records for publicly funded home care programs in Ontario. Physician visits were extracted from the Ontario Health Insurance Plan claims database, which contains information on inpatient and outpatient physician billings, including shadow billings for physicians in primarily capitated payment models. Emergency department visits were extracted from the National Ambulatory Care Reporting System, which captures all hospital and community-based ambulatory care in Ontario.

These data sets were linked using unique encoded identifiers and analyzed at ICES (formerly the Institiute for Clinical Evaluative Sciences). This study was granted an exemption from formal ethics review by the Hamilton Integrated Research Ethics Board as the use of data in this project was authorized under section 45 of Ontario's Personal Health Information Protection Act, which does not require review by a research ethics board.

\section{Participants}

Individuals receiving ongoing publicly funded home care in Ontario are regularly assessed with the
Resident Assessment Instrument for Home Care, a comprehensive clinical assessment. ${ }^{11}$ We selected all assessments for home care patients aged 19 years and older that were completed in Ontario from October 1, 2014 through September 30, 2016. If an individual was assessed more than once during the study, their most recent assessment was used. The assessment date was the index date for a 6 -month follow-up period. Patients receiving palliative home care were excluded from the cohort as they receive specialized nursing care.

\section{Case-Crossover Design}

The case-crossover design is a self-matched, case-only design in which cases act as their own controls. The design can be used to determine whether an event was caused by something that happened just before, and is immune to confounding by subject characteristics. ${ }^{12}$ For each subject in a case-crossover study, a case period during which the subject experiences the event of interest is matched with control periods (commonly the day(s) prior) during which the subject did not experience the event. An exposure of interest is measured immediately before each case and control period. ${ }^{13}$ The design enables researchers to determine whether there is transient association between the exposure and event by examining whether exposures were more likely to precede the case period rather than the control period(s).

In this study, the event of interest was an emergency department visit between 5:00 PM and midnight. We identified case periods by selecting all weekdays within 182 days following the index date on which a patient had a home nursing visit and visited the emergency department after 5:00 PM. Weekends and holidays were excluded as the availability of both home nursing and primary care differ substantially on weekends and weekdays. The earliest case period was utilized if a patient had more than 1 eligible case period during the follow-up period.

Control periods were identified by selecting all weekday evenings within the 7 days preceding the case period in which the patient did not have an emergency department visit. Control periods were matched to the case period for each patient. The exposure measured before each case or control period was whether a routine, prescheduled home nursing visit was received during the day. The identification and matching of case and control periods was performed separately for all emergency department visits and for the subset of visits that were not admitted to hospital. The nonadmitted visits represented less-urgent cases in which we expected both a stronger overall association and stronger effect modification between home nursing visits and same-day emergency department use. 


\section{Access to After-Hours Primary Care}

We operationalized access to after-hours primary care at both the patient and practice level. In Ontario, primary care physicians can receive premiums for services provided outside of traditional office hours. We used after-hours premium billing codes (Supplemental Appendix 2, https://www.AnnFamMed.org/ content/18/5/406/suppl/DC1/) to identify primary care visits that occurred after 5:00 PM on weekdays either in a physician's office or in a patient's home. These codes included care that took place during scheduled clinic hours that were after 5:00 PM and unscheduled care for urgent issues that took place after clinic hours.

The patient-level measure was based on an individual's use of after-hours primary care in the year preceding the case period and was split into 2 categories, any care vs no care. This definition is rooted in the concept that historical utilization provides evidence of health system access, and that past use of ambulatory care is a strong predictor of subsequent use. ${ }^{14}$ Since lack of utilization does not necessarily indicate lack of access, we also created a measure to capture the relative amount of after-hours care provided by a patient's primary care practice. Following the approach of Glazier et $\mathrm{al}_{1}{ }^{15}$ we calculated the proportion of all weekday care provided by a patient's primary care practice that could be identified as after-hours care. This was measured using visits of Ontario residents aged 65 years and older during the year in which the case occurred. As there was no theoretical basis for any specific cut points in this continuous measure, we categorized the practice-level provision of after-hours care by quartiles.

\section{Effect Modification}

The objective of this study was to examine the potential modifying effect of access to after-hours primary care on the increased risk of same-day emergency department visits following home nursing visits, ie, whether patients with greater access to after-hours primary care have a lower risk of an emergency department visit following a home nursing visit compared with patients with less access. This was accomplished by using the case-crossover design to estimate the effect of home nursing visits on same-day emergency department visits, and then testing the interaction between access to after-hours care and home nursing visits. This approach has the inherent robustness of case-crossover design to general confounding but is potentially at risk of bias if there were effect modifiers unaccounted for that correlated with after-hours care.

\section{Statistical Analysis}

The association between home nursing visits and same-day emergency department visits was estimated with conditional logistic regression, where each set of within-patient matched case and control periods formed separate strata. Effect modification by access to afterhours primary care was examined using an interaction term approach. Analysis was performed separately for all emergency department visits and for the less urgent subset of visits that were not admitted to hospital. We reported the odds ratios and $95 \%$ CIs of the association between home nursing and emergency department visits overall and within each category of the effect modifiers, as well as the $P$ value of the interaction term.

\section{Sensitivity Analysis}

We repeated the analyses including additional potential effect modifiers that may be correlated with access to after-hours care. These other modifiers were chosen based on previous research ${ }^{8}$ and included primary care practice type, rurality, activities of daily living, hierarchy score (a measure of function impairment), ${ }^{16}$ and presence of a live-in caregiver. We reported the odds ratio and $95 \%$ CIs of the association between home nursing and emergency department visits within each category of access to after-hours care, fixing the other effect modifiers at their mode (most frequent) value for categorical variables and mean value for continuous variables.

\section{RESULTS}

The final cohort contained 11,840 home care nursing patients with an emergency department visit who contributed a case period to the analysis. These case periods were matched to 55,210 control periods. For emergency department visits that did not result in hospital admission, there were 6,703 patients with case periods matched to 31,538 control periods. Patients contributing case periods to the analysis had a median age of 77 years and were balanced between males and females (Table 1). Just under one-half (46\%) needed at least limited assistance with activities of daily living and over one-half (54\%) had at least a mild cognitive impairment. Nearly all $(88 \%)$ of the patients had 5 or more concurrent medications, $35 \%$ were at least occasionally incontinent, and over $40 \%$ had a fall recently.

\section{Main Effect: Association Between Nursing Home Visits and Same-Day After-Hours Emergency Department Use}

Similar to previous work, receiving a home nursing visit was significantly associated with a greater likelihood of a same-day after-hours emergency department visit $(\mathrm{OR}=1.28 ; 95 \% \mathrm{CI}, 1.22-1.35)$ with a slightly larger effect for visits not admitted to hospital $(\mathrm{OR}=1.34 ; 95 \% \mathrm{CI}, 1.26-1.43)$ (Figure 1). 
Table 1. Characteristics of Home Care Patients

\begin{tabular}{|c|c|c|c|}
\hline Patient Characteristics & $\begin{array}{l}\text { All Patients } \\
(\mathrm{n}= \\
11,840) \\
\text { No. (\%) }\end{array}$ & $\begin{array}{l}\text { History of } \\
\text { After-Hours } \\
\text { Care Use }(n= \\
2,550) \text {, No. (\%) }\end{array}$ & $\begin{array}{l}\text { No History of } \\
\text { After-Hours } \\
\text { Care Use }(n= \\
9,290), \text { No. }(\%)\end{array}$ \\
\hline \multicolumn{4}{|l|}{ Demographics } \\
\hline Age, median (IQR), y & $77(65-86)$ & $77(66-87)$ & $76(64-85)$ \\
\hline Sex, female & $6,048(51)$ & $1,356(53)$ & $4,692(51)$ \\
\hline Living alone & $4,432(37)$ & $886(35)$ & $3,546(38)$ \\
\hline \multicolumn{4}{|l|}{ Health } \\
\hline \multicolumn{4}{|l|}{ ADL impairment ${ }^{\mathrm{a}}$} \\
\hline Independent/Supervision & $6,345(54)$ & $1,208(47)$ & $5,137(55)$ \\
\hline Limited/Extensive & $3,624(31)$ & 774 (30) & $2,850(31)$ \\
\hline Maximal/Dependent & $1,871(16)$ & $568(22)$ & $1,303(14)$ \\
\hline \multicolumn{4}{|l|}{ Cognitive impairment $\mathrm{t}^{\mathrm{b}}$} \\
\hline Intact/Borderline intact & $5,438(46)$ & $1,117(44)$ & $4,321(47)$ \\
\hline Mild/Moderate & $5,687(48)$ & $1,213(48)$ & $4,474(48)$ \\
\hline Severe & $715(6)$ & $220(9)$ & $495(5)$ \\
\hline \multicolumn{4}{|l|}{ Frailty index } \\
\hline Robust (0-0.19) & $2,420(20)$ & $450(18)$ & $1,970(21)$ \\
\hline Pre-frail (0.2-0.29) & $3,106(26)$ & $593(23)$ & $2,513(27)$ \\
\hline Frail $(\geq 0.3)$ & $6,314(53)$ & $1,507(59)$ & $4,807(52)$ \\
\hline Depressive symptoms & $6,615(56)$ & $1,477(58)$ & $5,138(55)$ \\
\hline Aggressive behaviors & $1,144(10)$ & $276(11)$ & $868(9)$ \\
\hline Fall in last 90 days & $4,823(41)$ & $1,008(40)$ & $3,815(41)$ \\
\hline Bladder incontinence & $4,147(35)$ & $1,009(40)$ & $3,138(34)$ \\
\hline Current medications $(\geq 5)$ & $10,399(88)$ & $2,265(89)$ & $8,134(88)$ \\
\hline Dementia & $1,832(15)$ & $445(17)$ & $1,387(15)$ \\
\hline \multicolumn{4}{|l|}{ Clinical need indicators } \\
\hline Wound care & $5,338(45)$ & $1,177(46)$ & $4,161(45)$ \\
\hline Intravenous medications & $1,116(9)$ & $206(8)$ & $910(10)$ \\
\hline Indwelling urinary catheter & $2,029(17)$ & $422(17)$ & $1,607(17)$ \\
\hline Congestive heart failure & $2,262(19)$ & $498(20)$ & $1,764(19)$ \\
\hline $\begin{array}{l}\text { Chronic obstructive pulmo- } \\
\text { nary disease }\end{array}$ & $3,096(26)$ & $652(26)$ & $2,444(26)$ \\
\hline \multicolumn{4}{|c|}{$A D L=$ activities of daily living; $I Q R=$ interquartile range. } \\
\hline \multicolumn{4}{|c|}{$\begin{array}{l}\text { a ADL Hierarchy Scale: Includes personal hygiene, locomotion, eating, and toileting. } \\
\text { b Cognitive performance scale } \\
\text { 'Scores on the frailty index range from } 0 \text { to } 1 \text {, where } 0 \text { represents no health deficits and } 1 \text { represents all pos- } \\
\text { sible health deficits. }\end{array}$} \\
\hline
\end{tabular}

who were not rostered to a primary care physician. Among the remaining cases, the quartile cut points for the practice-level proportion of care that was afterhours care were $3.2 \%, 5.6 \%$, and $9.4 \%$. While the observed associations in the second and fourth quartiles were lower than the first, we did not observe a monotonic trend toward a smaller risk of a same-day after-hours emergency department visit (Figure 1).

\section{Sensitivity Analysis}

Including additional effect modifiers increased the magnitude of the modifying effects of afterhours care and increased the standard errors of the main nursing effect but not the interaction effects. The result was greater significance for the interaction effects along with wider stratumspecific CIs of the main nursing effect (Figure 2).

\section{DISCUSSION}

We found that home care patients were more likely to visit the emergency department after 5:00 PM on the same day as a nursing visit, but that this association was smaller among patients who had a history of after-hours primary care use. This effect modification was stronger for the less-urgent set of emergency department vis-

\section{Effect Modification: Access to After-Hours Primary Care}

Just over one-fifth (21.5\%) of patients had least 1 after-hours weekday primary care visit in the previous year. These patients had a smaller increase in the risk of a same-day after-hours emergency department visit $(\mathrm{OR}=1.18 ; 95 \% \mathrm{CI}, 1.06-1.30)$ compared with patients with no after-hours care $(\mathrm{OR}=1.31 ; 95 \% \mathrm{CI}$, $1.25-1.39)$. The difference in risk was noticeably larger among emergency department visits not admitted to hospital $(\mathrm{OR}=1.11 ; 95 \% \mathrm{CI}, 0.97-1.28$ vs $\mathrm{OR}=1.41$; 95\% CI, 1.31-1.51) (Figure 1).

The practice-level provision of after-hours primary care could not be calculated for 771 patients $(6.5 \%)$ its that did not result in a hospital admission. We did not find a consistent effect related to the practice-level provision of after-hours primary care.

Some cross-sectional and survey studies have suggested that an increase in access to primary after-hours care could reduce emergency department visits ${ }^{4,17-19}$ while other cohort studies and systematic reviews of interventions found no reduction. ${ }^{5,6}$ Our study is novel in that it tests the effectiveness of after-hours primary care in terms of its ability to reduce the risk of an emergency department visit following an event known to increase risk and does so within a selfmatched study design. In contrast to other studies, our study does not seek to determine whether after-hours 
primary care can reduce emergency department use overall, but rather if it can prevent visits that would otherwise occur shortly after a home nursing visit.

Our results suggest that better access to afterhours primary care could prevent less-urgent emergency department visits following home nursing visits. While our study only examines the ability of after-hours primary care to reduce a short-term increased risk of an emergency department visit, the findings represent a novel contribution to the frequently conflicting literature on this topic. Reducing less-urgent emergency department visits is a priority of health systems globally as a means of improving continuity of care and reducing adverse outcomes such as emergency department-associated infections or delirium. ${ }^{20,21}$ Consequently, governments around the world have put policies in place to increase the availability of after-hours primary care. ${ }^{22}$ While the effectiveness of these policies have been called into question, ${ }^{5}$ our results contribute evidence to the argument that there is substantial benefit. Increasing the provision and use of after-hours primary care, at individual practices or system wide, through policy tools such as regulations or incentives could yield benefits for complex, community-dwelling older adults such as home care patients.

While our results suggest that expanding access to after-hours primary care would be beneficial, they also raise more questions. When examining the practicelevel provision of after-hours care, we did not observe a consistent dose-response relationship that is typical of causal effects. This lack of consistency at the practice level may be due to the importance of patient-level factors such as preference and awareness of the availability of after-hours primary care. In addition, we observed a smaller, more tenuous, modification of risk among all emergency department visits compared with the lessurgent subset of visits. Although this aligns with our original hypothesis, it raises questions as to what type and how many emergency department visits can be prevented with better access to after-hours primary care. Future research should further examine the influence of practicelevel measures of after-hours care provision on emergency department use as well as the types of emergency department visits that can be most successfully diverted to after-hours primary care.

\section{Limitations}

Our study has a number of strengths, including use of population-based data and a selfmatched design, but also notable weaknesses. First, there is no precise method to determine which emergency department visits could be more appropriately seen in primary care. Additionally, as the case-crossover study

$\mathrm{ED}=$ emergency department; Q1 = Quartile 1; Q2 = Quartile 2; Q3 = Quartile 3; Q4 = Quartile 4. 
is a case-only design, only the patients who visited the emergency department after 5:00 PM contributed to the analysis. Also, as we used claims-based data, our measures of access to after-hours primary care are utilizationbased proxies. Finally, our study examines a very specific question concerning emergency department visits following home care nursing visits. This is a strength in that it allows for clarity in interpretation, but also means that our findings cannot be generalized to overall emergency department use. It may be reasonable to assume, however, that our findings generalize to emergency department visits following visits with other health care clinicians when emerging health issues may be identified.

\section{Conclusion}

Home care patients were more likely visit an emergency department following a home nursing visit, but patients with a history of after-hours primary care use had a smaller risk, particularly for less-urgent emergency department visits. This suggests better access to after-hours primary care can reduce certain specific, shortterm risks of less-urgent emergency department visits. These findings contribute evidence to the literature supporting the benefits of increasing the availability of after-hours primary care.

To read or post commentaries in response to this article, see it online at https://www.AnnFamMed.org/content/18/5/406.

Key words: after-hours care; health services for the aged; home care services; primary health care

Submitted September 25, 2019; submitted, revised, January 29, 2020; accepted February 5, 2020.

Funding support: This study was funded by a grant from the Canadian Institutes for Health Research (148933). The opinions and conclusions expressed in this article are those of the authors and do not necessarily reflect the views of the contributing centers or funder.

Previous presentation: A poster entitled "Primary care effect modification of a transient association between home care nursing and sameday emergency department visits" was presented at the Canadian Asso- ciation for Health Services and Policy Research conference; May 29-31, 2019; Halifax, Canada.

Acknowledgments: The authors would like to thank Ahmad Rahim from ICES for his assistance with data curation. This study was supported by ICES, which is funded by an annual grant from the Ontario Ministry of Health and Long-Term Care (MOHLTC). The opinions, results and conclusions reported in this paper are those of the authors and are independent from the funding sources. No endorsement by ICES or the Ontario MOHLTC is intended or should be inferred. Parts of this material are based on data and/or information compiled and provided by the Canadian Institute for Health Information (CIHI). The analyses, conclusions, opinions and statements expressed in the material are those of the authors, however, and not necessarily those of $\mathrm{CIHI}$.

Supplemental materials: Available at https://www.AnnFamMed. org/content/18/5/406/suppl/DC1/. 


\section{References}

1. Smits $M$, Rutten $M$, Keizer $E$, Wensing $M$, Westert $G$, Giesen P. The development and performance of after-hours primary care in the Netherlands: a narrative review. Ann Intern Med. 2017;166(10):737742. 10.7326/M16-2776.

2. Hwang $U$, Morrison RS. The geriatric emergency department. J Am Geriatr Soc. 2007;55(11):1873-1876. 10.1111/j.1532-5415.2007. 01400.x.

3. van Uden CJ, Winkens RA, Wesseling G, Fiolet HF, van Schayck OC, Crebolder HF. The impact of a primary care physician cooperative on the caseload of an emergency department: the Maastricht integrated out-of-hours service. J Gen Intern Med. 2005;20(7):612-617.

4. Posocco A, Scapinello MP, De Ronch I, et al. Role of out of hours primary care service in limiting inappropriate access to emergency department. Intern Emerg Med. 2018;13(4):549-555. 10.1007/ s11739-017-1679-8.

5. Kiran T, Moineddin R, Kopp A, Frymire E, Glazier RH. Emergency department use and enrollment in a medical home providing afterhours care. Ann Fam Med. 2018;16(5):419-427. 10.1370/afm.2291.

6. Ismail SA, Gibbons DC, Gnani S. Reducing inappropriate accident and emergency department attendances: a systematic review of primary care service interventions. Br J Gen Pract. 2013;63(617): e813-e820. 10.3399/bjgp13X675395.

7. National Prevention Council. Healthy Aging in Action: Advancing the National Prevention Strategy. Washington, DC: US Department of Health and Human Services; 2016. https://www.ncbi.nlm.nih.gov/ books/NBK538955/.

8. Jones A, Bronskill SE, Agarwal G, Seow H, Feeny D, Costa AP. The primary care and other health system use of home care patients: a retrospective cohort analysis. CMAJ Open. 2019;7(2):E360-E370. 10.9778/cmajo.20190038.

9. Jones A, Schumacher C, Bronskill SE, et al. The association between home care visits and same-day emergency department use: a case-crossover study. CMAJ. 2018;190(17):E525-E531. 10.1503/ cmaj.170892.

10. Canadian Nurses Association (CAN). Optimizing the role of nursing in home health. https://cna-aiic.ca/ /media/cna/page-content/ pdf-en/optimizing_the_role_of_nursing_in_home_health_e. pdf?la=en. Published May 30, 2013. Accessed Jul 20, 2017.
11. Canadian Institute for Health Information. Home Care Reporting System Data Users Guide 2017-2018. Ottawa, Ontario, CA: Canadian Institute for Health Information; 2019. https://secure.cihi.ca/free_ products/HCRS-external-data-users-guide-2017-2018-en-web.pdf.

12. Maclure M, Mittleman MA. Should we use a case-crossover design? Annu Rev Public Health. 2000;21(1):193-221. 10.1146/annurev. publhealth.21.1.193.

13. Maclure M. The case-crossover design: a method for studying transient effects on the risk of acute events. Am J Epidemiol. 1991;133(2): 144-153.

14. Jordan K, Ong BN, Croft P. Previous consultation and self reported health status as predictors of future demand for primary care. J Epidemiol Community Health. 2003;57(2):109-113. 10.1136/ jech.57.2.109.

15. Glazier RH, Klein-Geltink J, Kopp A, Sibley LM. Capitation and enhanced fee-for-service models for primary care reform: a population-based evaluation. CMAJ. 2009;180(11):E72-E81. 10.1503/ cmaj.081316.

16. Morris JN, Fries BE, Morris SA. Scaling ADLs within the MDS. J Gerontol A Biol Sci Med Sci. 1999;54(11):M546-M553.

17. O'Malley AS. After-hours access to primary care practices linked with lower emergency department use and less unmet medical need. Health Aff (Millwood). 2013;32(1):175-183. 10.1377/ hlthaff.2012.0494.

18. Harris MJ, Patel B, Bowen S. Primary care access and its relationship with emergency department utilisation: an observational, crosssectional, ecological study. Br J Gen Pract. 2011;61(593):e787-e793.

19. Wong W-B, Edgar G, Liddy C, Vaillancourt C. Can after-hours family medicine clinics represent an alternative to emergency departments? Survey of ambulatory patients seeking after-hours care. Can Fam Physician. 2009;55(11):1106-1107.e4.

20. Quach C, McArthur M, McGeer A, et al. Risk of infection following a visit to the emergency department: a cohort study. CMAJ. 2012; 184(4):E232-E239. 10.1503/cmaj.110372.

21. Émond M, Grenier D, Morin J, et al. Emergency department stay associated delirium in older patients. Can Geriatr J. 2017;20(1):10-14. doi:10.5770/cgj.20.246

22. Berchet $C$, Nader $C$. The organisation of out-of-hours primary care in OECD countries. Paris, France: OECD Publishing; 2016. doi: https://doi.org/10.1787/5jlr3czbqw23-en 